\title{
( $\mathrm{BiPb})-\mathrm{Sr}-(\mathrm{CaCd})-\mathrm{Cu}-\mathrm{O}$ 系的超导电性
}

\author{
王晓临 蒋民华王弘董胜明于文涛尚淑霞王、卓 \\ (山东大学晶体材料研究所, 济南 250100)
}

\section{关䇥词 Bi 系超导体、2212 相、单斜相、105K 零电阻}

自从Bi系超导体发现以来以, 人们已经确定了至少三个超导相 (2201、2212、2223 相), 各 相每半个晶胞中含 $1 、 2 、 3$ 层铜层, 其电阻温度正比于单胞中铜氧层的数目, 分别为 7 、 $85 、 110 \mathrm{~K}$. 为了获得更高转变温度的超导相, 中国科技大学用少量 $\mathrm{Sb}$ 替代 $\mathrm{Bi}$ 发现了零电 阻温度大于 $132 \mathrm{~K}$ 的超导现象 ${ }^{[n}$, 此结果陆续被几个研究小组重复 ${ }^{[3-\Im]}$, 有的样品甚至高于 160 K. 但至今关于 $132 \mathrm{~K}$ 高温超导相的结构或形成的可能机制尚未确定. 最近, 我们采用直接烧 结一空气淬火的一次烧结工艺确定了含 4 层 $\mathrm{Cu}-\mathrm{O}_{2}$ 层的 2234 相的零电阻温度为 $95 \mathrm{~K}^{[6]}$, 这 说明过多的 $\mathrm{Cu}-\mathrm{O}_{2}$ 层反而导致超导电性的减弱. $132 \mathrm{~K}$ 高温超导相不可能是含更多 $\mathrm{Cu}-\mathrm{O}_{2}$ 层的超导相, 只可能是一种未知新相或许也可能与其它非超导相相关. 在最初的含有 $132 \mathrm{~K}$ 相的 $X$ 光衍射图中只观察到了 $2 \theta \sim 30^{\circ}$ 附近两个可能与之相联系的未知峰 ${ }^{[2]}$. 最近中国科技 大学叻确定了此二峰是单斜相 $\mathrm{Bi}_{4} \mathrm{Sr}_{4} \mathrm{Ca}_{4} \mathrm{CuO}$, 的两个特征峰, 并确定了该单斜相具有绝缘体 形为 ${ }^{1}$. 我们注意到与 $T_{\mathrm{co}}$ 为 $132 \mathrm{~K}$ 相关的样品的 X 光衍射谱图中在 $2 \theta \sim 30^{\circ}$ 附近存在属于 单斜相的两个特征峰 ${ }^{[2.823)}$, 且若样品中只有 2223 相与单斜相共存, 而无其它相, 象 2201,2212

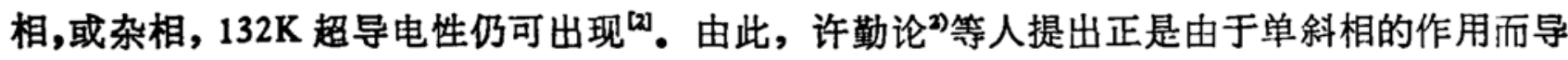
致了 $132 \mathrm{~K}$ 超导性. 目前, 对此也未定论以. 我们设想如果单斜相只与 2212 相或 2201 相共存 是否也能提高样品的电阻温度. 若能提高, 无疑会对 $132 \mathrm{~K}$ 超导电性起因的揭示有很大的 意义. 本文报道, 采用直接烧结一空气淬火的一次烧结工艺, 在掺 $\mathrm{Cd}$ 的 $\mathrm{Bi}$ 系陶瓷样品中发 现若只有 2212 相与单斜相共存样品的零电阻可高达 $105 \mathrm{~K}$.

\section{一、实 验}

现已发现, Bi 系中掺 Sb 或 Cd 对超导相的形成有不同的作用,少量 Sb 有利于 2223 相 或 2234 相的生长 ${ }^{[6,9]}$, 而 $\mathrm{Cd}$ 的作用则是易于生长 2212 相. 因此, 我们在 $\mathrm{Bi}$ 系中掺 $\mathrm{Cd}$ 并 选择合理的烧结条件制备出单斜相只与 2212 相共存的样品. 样品制备方法采用直接烧结-空 气淬火的一次烧结工艺 ${ }^{[6]}$. 分析纯的 $\mathrm{Bi}_{2} \mathrm{O}_{3} 、 \mathrm{SrCO}_{3} 、 \mathrm{CaCO}_{3} 、 \mathrm{CdO} 、 \mathrm{PbO} 、 \mathrm{CuO}$ 按名义组分 $(\mathrm{BiPb})_{2} \mathrm{Sr}_{2} \mathrm{C}_{2} \mathrm{Cd}_{2} \mathrm{Cu}_{3} \mathrm{O}_{y}(x-0.1-1)$, 配比、称重、混匀. 本文只给出两个代表性样品的结 果, 一是掺 $\mathrm{Cd}_{0.1}$, 烧结条件为在 $825^{\circ} \mathrm{C}$ 烧结 $144 \mathrm{~h}$; 另一是掺 $\mathrm{Cd}_{0.3}$, 烧结条件为在 $830^{\circ} \mathrm{C}$ 烧 结 $120 \mathrm{~h}$. 样品电阻测量采用标准的四引线方法, 测量电流 $1 \mathrm{~mA}$, 电阻温度计误差 $\pm 0.1 \mathrm{~K}$, 用

本文 1990 年 9 月 27 日收到. 1991 年 5 月 16 日收到焦改稿.

1) Wang, X. D., Meng, G. Y. et al., Solid State Commun., in print.

2) Xiu Qinlun, Meng Guangyao et al., C-MRS (1990) Proceeding, in print. 


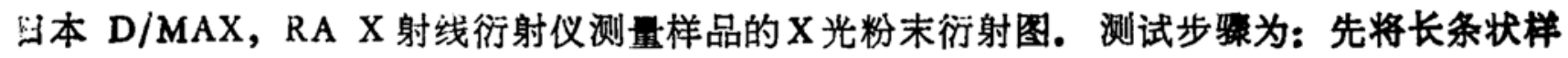
品医钢电极测电阻, 然后将测完电阻后的同一样品研碎做 $\mathbf{X}$ 光结构分析.

\section{二、结果和讨论}

图 1 是掺 $\mathrm{Cd}_{0.3}$ 样品的 $R-T$ 图, 零电阻为 $80 \mathrm{~K}$. 图 2(a) 是它的 $\mathrm{X}$ 光衍射图. 可以看出, 该样品几乎是单相的 2212 相, 2201 相极少. 图 3 为掺 $\mathrm{Cd}_{0.1}$ 样品的 $R-T$ 曲线. 阻为 105 K. 图 2(b) 为该样品的 X 光衍射谱图. 图中显示该样品中无 2223 相, 2201 相极少可忽略, 只有一个超导相 2212 相. 在 $2 \theta \sim 30^{\circ}$ 附近有两个衍射峰其 $d$ 值为 $2.99 \AA$ 和 $2.94 \AA$ (图中箭头 所示), 它们正好是属于单斜相的两个特征峰, 标志着样品中单斜相与 2212 相共存. 因为 单斜相已确定为绝缘体 ${ }^{1)}$, 并且对于无掺杂的 2212 相的零电阻温度从目前文献上来看一般在 80 - $90 \mathrm{~K}$ 范围内变化 ${ }^{[11,12]}$, 不可能高于 $95 \mathrm{~K}^{[13]}$; 另外, 掺 $\mathrm{Cd}$ 以后, 由于 $\mathrm{Cd}$ 会进入晶胞而代 替 $\mathrm{Ca}$, 且因 $\mathrm{Cd}^{2+}$ 半径小于 $\mathrm{Ca}^{2+}$ 半径, 从而导致了晶胞参数 $c$ 轴的减小, 超导电性降低 ${ }^{(10)}$. 我们也已经发现随 $\mathrm{Cd}$ 的加人使得 2212 相的零电阻温度从 $85 \mathrm{~K}$ 下降到 $77 \mathrm{~K}$ 以下年1 . 这说 明掺 Cd 本身并不能提高 2212 相的零电阻温度,反而导致 $T_{c o}$ 的下降. 比较图 1、图 3 与图 $2(a) 、(b)$, 无单斜相的 2212 相样品的 $T_{\text {co }}$ 为 $80 \mathrm{~K}$, 而与单斜相共存的 2212 相样品 $T_{\text {co }}$ 为 $105 \mathrm{~K}$, 形成鲜明的对比. 我们在掺 $\mathrm{Sb} .1$ 的 $\mathrm{Bi}$ 系陶瓷样品中也发现了单斜相只与 2223 相

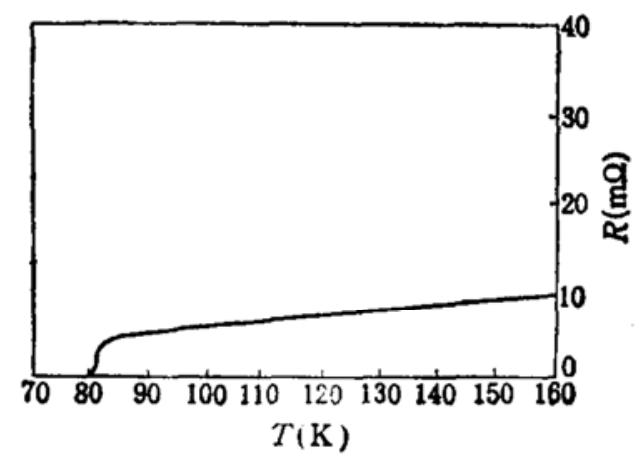

图 $1(\mathrm{Bi}, \mathrm{Pb})_{2} \mathrm{Sr}_{2} \mathrm{Ca}_{2} \mathrm{Cd}_{0,3} \mathrm{Cu}_{3} \mathrm{O} y$ 的 $R-T$ 曲线 绕结温度 $830^{\circ} \mathrm{C}$, 时间 $120 \mathrm{~h}$

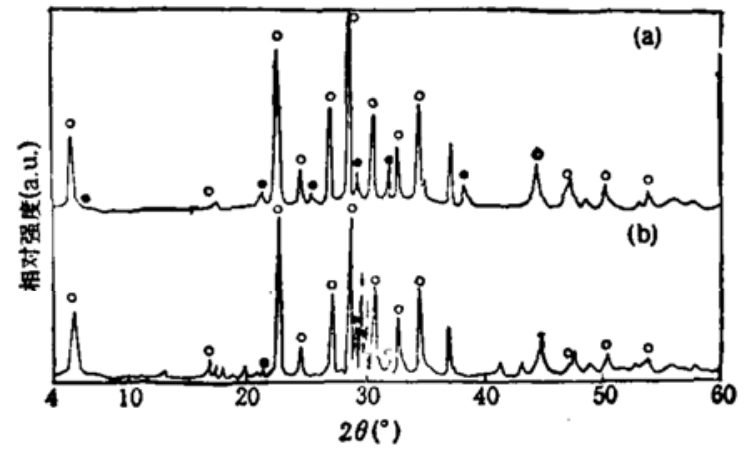

图 2 (a)、(b) 分别为 $\mathrm{BiPbSr}_{2} \mathrm{Ca}_{2} \mathrm{Cd}_{0.3}, \mathrm{Cu}, \mathrm{Oy}$ 和 $(\mathrm{Bi} \mathrm{Pb})_{2} \mathrm{Sr}_{2} \mathrm{Ca}_{2} \mathrm{Cd}_{0.1} \mathrm{Cu}_{3} \mathrm{O} y$ 的 $\mathrm{X}$ 光谱图 ○代表 2212 相; - 代表 2201 相. 简头和无符号峰为单舴相

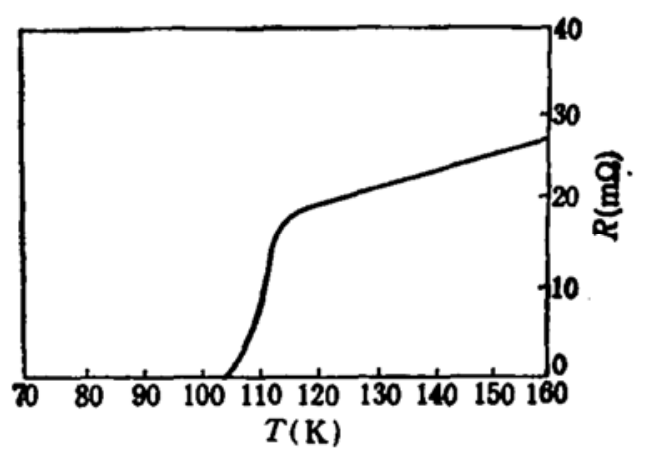

图 3 (Bi Pb) ${ }_{2} \mathrm{Sr}_{3} \mathrm{Ca}_{2} \mathrm{Cd}_{0.1}, \mathrm{Cu}_{3}, \mathrm{O} y$ 的 $R-T$ 曲线 烧结温度 $825^{\circ} \mathrm{C}$, 时间 $144 \mathrm{~h}$

1) 同上页街注. 
共存, 样品的电阻在 $148 \rightarrow 132 \mathrm{~K}$ 有陡降, 说明样品可能具有高于 $132 \mathrm{~K}$ 的超导电性, 若没有单 料相只有 2223 相则样品的 $T_{\mathrm{co}}$ 为 $108 \mathrm{~K}$. 从以上结果及分析来看, 单斜相与超导相的共存 确实可以提高样品的电阻温度, 并且我们认为单斜相对 2212 相及 2223 相超导电性的提高 有可能出于同一机理. 按照由于单斜相的作用使 2212 相样品的 $T_{\mathrm{co}}$ 从 80-90K 提高到 105 $\mathrm{K}$, 提高了. $15-25 \mathrm{~K}$, 粗略估算一下由于单斜相的作用对 2223 相样品 $T_{\mathrm{co}}$ 的提高值, 取无单 料相 2223 相样品的 $T_{\mathrm{co}}$ 为 $110 \mathrm{~K}$, 则其电阻可提高到 $125-135 \mathrm{~K}$ 这与实验结果相当吻 合. 对有 $132 \mathrm{~K}$ 超导性的样品的零电阻一般在 $125 \mathrm{~K}$ 或 $132 \mathrm{~K}$ 降为管 ${ }^{[4,5]}$.

进一步的研究, 象 2201 相与单斜相共存可否提高样品的零电阻温度; 究竟单斜相是如何 提高样品的 $T_{\mathrm{co}}$, 从微观机制如何解释, 以及由此可否确定高于 $132 \mathrm{~K}$ 超导电性的机制我们 正在做深人的工作.

\section{台考文献}

[ 1 ] Maeda, H., Tanaka, T. et al., Jpn. J. Appl. Phys., 27(1988), L209.

I 2 l Liu Hongbao, Cao liezhao et al., Solid State Commun, 69(1989), 8: 867.

(3) Chandrachood, M. R. and Mulla, I. S., Appl. Phys. Letr, 55(1989), 1472.

[4] Haung, C. J., Tseng, T. Y. et al., Solid State Commun, 72(1989), 6: 563.

[5] 贾国强、吴晓祖、㞭青云,物理学报,39(1990),8: 1310.

16 ] Wang Hong, Wang Xiaolin et al., Appl. Phys. Lent, 57(1990), 710.

I 7 ] Cheng, T. Z., Fan, C. G. et al., Superconductor Science and Technology, 3(1990), 87.

[ 8 ] Sastry, P. V. P. S. S. et al., Solid Staze Commun, 71(1989), 935.

[ 9 ] Konstantinov, K. et al, Z. Phys. B-Condensed Manter. 81(1990), 151.

f10] Wang Xiaolin, Wang Hong et al., Solid Staze Commun, 76(1990), 5: 675-677.

[11] 车广奾、梁故鬿等, 科学通报, 34(1989), 12: 895 .

[12] Wang Hong et al., J. Crystal Growth, 99(1990), 929.

[13] Ishida, T., Jpn. J. Appl. Phys, 27(1988), ¿2327.

[14] Yoshimura, M. et al., Jpn. J. Appl. Phys, 3(1989!, L424. 